\title{
BERNARD COTTRET
}

\section{LA RESTAURATION ANGLAISE COMME PAIX DE RELIGION, 1660-1688}

Contrairement aux espérances qu'elle a pu susciter en Angleterre, la restauration des Stuarts a accouché dans la douleur d'une société divisée. Fracture sociale? Fracture politique? Ou fracture religieuse? Quelles que fussent leur sociologie, ou leurs déterminations économiques profondes, les tensions s'exprimèrent essentiellement sur le mode religieux: l'Église nationale, que l'on aurait souhaitée une et indivisible, ne parvint pas à accueillir en son sein tous ses fils dispersés. L'Église d'Angleterre, "autrefois inclusive«, devint "persécutrice« et »coupa la nation en deux «'. Cela s'accompagna d'une intéressante évolution sémantique: le développement accéléré d'une conscience anglicane distincte au sein du protestantisme ${ }^{2}$. La situation est d'autant plus complexe outre-Manche que la Réforme s'y déroula sur au moins trois siècles ${ }^{3}$.

L'anglicanisme se chargea en quelques années d'un contenu doctrinal de plus en plus net ${ }^{4}$. Il reposait sur une ecclésiologie singulière, fondée sur le magistère des évêques, seuls habilités à ordonner des ministres ou à confirmer des laïcs. Il est intéressant de noter que le terme d'»épiscopalisme« est à l'heure actuelle en usage dans une partie du monde anglophone pour désigner la communion anglicane. Cette synonymie marque à l'évidence l'attachement des anglicans à une conception pyramidale des ministères là où presbytériens et indépendants congrégationalistes valorisaient les assemblées, associant pasteurs et laïcs.

L'anglicanisme, fruit de la Restauration? En un sens, en un sens seulement. Certes, le phénomène avait connu une longue période de gestation, entre la rupture avec Rome en 1536, l'adoption des »Trente-Neuf Articles« en 15631571, ou la traduction de la Bible de 1611. L'ecclesia anglicana comme

1 Joan THIRSK, The Restoration, Londres 1976, p. XVI.

2 Le séjour de plusieurs royalistes anglicans sur le continent participa aussi à cette prise de conscience en leur permettant de mettre le doigt sur ce qui les séparait des protestants français par exemple. Le futur évêque de Durham, John Cosin, expliquait à ses coreligionnaires exilés anglicans qu'ils pouvaient prendre la sainte cène dans les cultes réformés à condition que le ministre dise clairement, avec toute la déférence requise: »Prenez, mangez les viandes sacrées de notre Seigneur« (Bernard COTTRET, The Huguenots in England, Cambridge 1991, p. 173).

3 Voir en particulier Nicholas TYACKE (dir.), England's Long Reformation, 15001800 , Londres 1998.

4 Alain JoBLIN, Jacques Sys (dir.), L'identité anglicane, Arras 2004. 
l'ecclesia gallicana pouvaient s'enorgueillir d'un passé très ancien; mais si l'une aboutit à une confession distincte, l'anglicanisme, le gallicanisme d'ancien régime ne rompit pas totalement avec le catholicisme romain. Désormais, l'ecclesia anglicana ne relevait plus de la géographie; l'Église en Angleterre était progressivement devenue l'Église d'Angleterre, une confession consciente d'elle-même, de plus en plus amenée à afficher son identité face aux autres Églises protestantes, issues de la Réforme magistérielle, qu'il s'agit des réformés ou des luthériens du continent.

Tout tient cependant à ce paradoxe que l'anglicanisme n'effectua son unification qu'en expulsant dans ses marges un nombre important de fidèles, qualifiés désormais de »dissenters« ou encore de non-conformistes. Le dissent fut l'un des fruits amères de la Restauration. Désormais, aux côtés de l'Église officielle, un nombre assez important d'Anglais adoraient Dieu à leur façon, dans ces chapels ou ces meeting-houses où presbytériens, indépendants, quakers ou baptistes, rejoints au XVIII ${ }^{\mathrm{e}}$ siècle par les méthodistes, célébraient officieusement leurs cultes.

Il fallut attendre 1689 et la loi généralement appelée, de façon certes discutable, le Toleration Act pour qu'ils pussent le faire librement. D'où la périodisation retenue ici qui s'attachera à deux moments forts de l'histoire britannique: le retour des Stuarts en 1660 , la Glorieuse Révolution de 1688 . À sa façon discrète, cette dernière peut d'ailleurs être interprétée également comme une restauration, comme nous le démontrerons plus avant.

\section{Concorde ecclésiastique et confessionnalisation}

Nous emprunterons à l'historiographie allemande ce souci de la confessionnalisation, qui renvoie à des termes distincts outre-Rhin: "Konfessionsbildung « et "Konfessionalisierung «. Les deux restent distincts, même si on a eu tendance à les rendre par le même terme en français ${ }^{5}$. "Konfessionsbildung «, dans l'espace germanique, décrit essentiellement le face à face entre catholiques et protestants, amenés de part et d'autre à approfondir leurs convictions ${ }^{6}$. Il s'agit donc d'une confrontation entre Eglises différentes. »Konfessionalisierung «, par contre, définit un phénomène interne à chaque Église,

5 Thierry WANEGFFelen, Les chrétiens face aux Églises dans l'Europe moderne, dans: Nouvelle Revue du Seizième Siècle 11 (1993), p. 37-53. Dans le cas particulier de l'Angleterre, voir Caroline J. LITZENBERGER, Defining the Church of England, dans: ID., Susan WABUDA (dir.), Belief and Practice in Reformation England, Aldershot 1998, p. 137-138.

6 Ernst Walter ZEEDEN, Konfessionsbildung: Studien zur Reformation, Gegenreformation und katholischen Reform, Stuttgart 1985. 
amenée à imposer à ses fidèles ses normes doctrinales, organisationnelles ou éthiques ${ }^{7}$. Or, la singularité de la situation anglaise, dans les années 1660 , a été précisément de confondre les deux phénomènes: il s'agissait autant d'imposer l'anglicanisme aux anglicans, si l'on peut dire, que de définir une nouvelle frontière religieuse, excluant de facto les insoumis. La rivalité confessionnelle classique entre Anglais, membres de l'Église nationale, et catholiques romains, présentés comme les sujets d'une puissance étrangère, se doublait désormais d'une autre barrière, séparant anglicans et dissenters. Le protestantisme lui-même avait éclaté; si le pays avait connu depuis la période élisabéthaine sa frange séparatiste, se réunissant en marge des paroisses autorisées, le phénomène devenait soudain plus massif, encore qu'il soit difficile de parvenir à des chiffres précis. Mais l'on peut estimer qu'environ $10 \%$ des Anglais, sur une population estimée à environ 5500000 habitants n'appartenaient pas à l'Église nationale, ou du moins ne se rendaient que de façon épisodique dans les paroisses autorisées. Sur ces $10 \%$, une minorité de catholiques, environ $1 \%$, toujours soupçonnée de trahison.

La Restauration se termina assez mal, en dépit de débuts prometteurs. À la veille même de son débarquement, Charles II avait donné en avril 1660 un texte manifeste, connu sous le nom de "Déclaration de Breda«, du nom de la ville de Hollande où se trouvait le monarque, en quête de son trône. Charles "par la grâce de Dieu, roi d'Angleterre, d'Écosse, de France et d'Irlande, défenseur de la foi, [etc.]《 s'adressait à tous ses bons sujets, de tout état et de toute qualité. Il déplorait les troubles et la confusion qui s'étaient abattus sur le royaume et évoquait le désir qu'éprouvaient tous les hommes de bonne volonté de voir guérir les blessures de la guerre civile et de l'interrègne. Il rappelait que Dieu et la nature lui rendaient ses droits, en évitant toute effusion de sang, et promettait son pardon à tous ceux qui le méritaient. Formule à dessein évasive, mais on ne saurait a priori douter ni de son sérieux ni de son réalisme. Déjà face au Parlement dans les années 1640, la couronne avait toujours invoqué son attachement à la paix civile, en présentant ses adversaires comme des trublions et des boutefeux. La monarchie souhaitait l'amour sincère des Anglais, en écartant la crainte de la punition. Le regret sincère de leurs fautes et non la peur du châtiment devaient animer désormais tous les sujets. On retrouvera bien ici en filigrane la théologie de la contrition. "Toute occasion de discorde, de séparation ou de division partisane devait être abolie«.

Certes, l'absence de charité et les passions avaient amené l'expression de "nombreuses opinions sur le plan religieux «, entraînant la constitution de partis et le développement de l'animosité entre les hommes. Ainsi la diver-

7 Heinz ScHILling (dir.), Die reformierte Konfessionalisierung in Deutschland. Das Problem der »Zweiten Reformation«, Gütersloh 1986. 
gence confessionnelle était-elle clairement présentée comme la cause de l'émergence de partis. La religion, clé de la politique? Cette étiologie, peutêtre contestable, de la crise révolutionnaire et de son développement revêtait d'importantes conséquences pour l'avenir: si la division religieuse constituait la cause de la division politique, comment ne pas en déduire que l'unité confessionnelle, la conformité ecclésiastique et la double suprématie royale sur l'Église et sur l'État pouvaient seules garantir la paix civile? Et pourtant, tout en prêchant cette unanimité nationale, Charles II reconnaissait, d'une certaine façon, l'existence de divergences doctrinales, qui devaient se prêter à des échanges sans contrainte. Il garantissait ainsi à ses sujets la possibilité de dialoguer entre eux - "a freedom of conversation « - et proclamait son attachement à la liberté de conscience: "We do declare a liberty to tender consciences«. Nul ne devait être inquiété pour ses opinions en matière de religion, tant que la paix du royaume n'était pas menacée. Et Charles II de promettre, avec l'accord du Parlement, une politique d'indulgence à l'égard des consciences scrupuleuses, $»$ tender consciences $«$ en anglais ${ }^{8}$.

»Indulgence«: le mot était lâché. Il allait faire l'objet de multiples reprises ultérieures, tant par Charles II que par son frère Jacques II, roi effectif de 1685 jusqu'à son départ en décembre 1688. Quelle était cette coupable indulgence que des générations élevées dans la tradition de l'histoire whig reprochèrent aux Stuarts? Le mot provenait du latin indulgentia, décrivant une concession. La politique d'indulgence répondait à un objectif clairement avoué: Charles II promettait à la fois de rétablir l'unité religieuse du royaume, avec l'accord du Parlement, et de garantir, selon des modalités encore incertaines, la liberté de conscience. Ce programme en soi contradictoire reposait en réalité sur une axiomatique de la grâce royale, apparue de plus en plus clairement avec les années.

\section{L'oubli et le pardon}

Autre pièce de l'édifice, l'Acte sur l'indemnité et l'oubli, discuté au printemps 1660 et finalement adopté définitivement fin août ${ }^{9}$. Une poignée de régicides devaient payer pour les autres, le cadavre de Cromwell était exhu-

8 King Charles II. His declaration to all his loving subjects of the kingdom of England. Dated from his Court at Breda in Holland, the 4/14 of April 1660. And read in Parliament, May, 1. 1660. Together with his Majesties letter of the same date, to his Excellence the Lord General Monck, to be communicated to the Lord President of the Council of State, and to the officers of the army under his command, Edimbourg, C. Higgins, 1660.

9 »An Act of Free and General Pardon, Indemnity and Oblivion« (12 Car. II, c. 11). 
mé et pendu... mais Milton, un temps son secrétaire, un temps défenseur de l'exécution de Charles I ${ }^{\mathrm{er}}$, put poursuivre sa carrière poétique. Le 29 août, Charles II remerciait solennellement son Parlement d'avoir adopté ce texte garantissant »la sécurité« et »le bonheur« de tous. La »clémence«, expliquait le roi magnanime, était bien dans sa nature ${ }^{10}$. Mais il laissait clairement entendre qu'à l'avenir il saurait tuer dans l'œuf toute rébellion. En octobre s'ouvrait le procès des régicides, exécutés sans attendre à Charing Cross ou à Tyburn. Thomas Harrison, John Jones, Adrian Scroope, John Carew, Thomas Scot, et Gregory Clement, le révérend Hugh Peters, Daniel Axtel, et John Cook connurent le sort peu enviable qui s'abattait sur les coupables convaincus de lèse-majesté: ils furent pendus et sauvagement mutilés et découpés: Francis Hacker fut simplement pendu ${ }^{11}$. Trois autres devaient connaitre un sort analogue deux ans plus tard.

Sur cet arrière-plan répressif, l'acte de Pardon, d'Indemnité et d'Oubli frappe par sa tonalité philosophique. Il repose sur un soigneux dosage de mémoire et d'oubli, de ressentiment et de pardon. Charles II considérait avec wattention les longues périodes de trouble, de discorde et de guerre qui ont pesé sur ce royaume, ainsi que les châtiments et les punitions qui devraient échoir à nombre de ses sujets«. Il souhaitait

de tout cœur mettre un terme à toutes les controverses et à toutes les polémiques qui se sont élevées ou qui sont encore susceptibles de s'élever entre ses sujets, afin qu'aucun crime commis contre Sa Majesté ou contre le roi son père ne puisse être imputé à quiconque, en compromettant ainsi sa réputation, sa vie, sa liberté ou ses biens.

Il s'agissait bien d'enterrer définitivement "tous les germes de discorde future ou passée ${ }^{12}$. Singulière prescription de l'oubli, et qui n'est pas sans évoquer la teneur de l'édit de Nantes, ratifié plus de soixante ans plus tôt par Henri IV, grand-père maternel de Charles II $^{13}$.

Que la mémoire de toutes choses passées d'une part et d'autre, depuis le commencement du mois de mars 1585 jusqu'à notre avènement à la couronne, et durant les

10 His Majesties gracious speech to both Houses of Parliament, on the 29th. day of August 1660, Londres, J. Bill, C. Barker, 1660, p. 4.

11 An Exact and Impartial Accompt of the Indictment, Arraignment, Tryal, and Judgment (according to Law) of Twenty Nine Regicides, The Murtherers of His Late Sacred Majesty of Most Glorious Memory, Londres, A. Crook, E. Powell, 1660.

12 Anno Regni Caroli II. Regis Scotiae Angliae Franciae \& Hiberniae duodecimo at the Parliament begun at Westminster, the five and twentieth day of April, Anno Dom. 1660, in the twelfth year of the reign of our most Gracious Soveraign Lord Charles, by the grace of God, of England, Scotland, France and Ireland, King, defender of the faith, \&c., Londres, J. Bill, C. Barker, 1660. Sur le maintien d'un underground radical, voir R.L. GREAVES, Deliver Us from Evil, New York 1986.

13 Bernard COTTRET, 1598-1688: de l'édit de Nantes à la Glorieuse Révolution. Concorde, liberté de conscience, tolérance, dans: Antoine Capet, Jean-Paul PICHARDIE (dir.), La naissance de l'idée de tolérance, Rouen 1999, p. 3-28. 
autres troubles précédents et à l'occasion d'iceux, demeurera éteinte et assoupie, comme de chose non advenue; et ne sera loisible ni permis à nos procureurs généraux, ni autres personnes quelconques, publiques ni privées, en quelque temps, ni pour quelque occasion que ce soit, en faire mention, procès ou poursuite en aucune cour et juridiction que ce soit ${ }^{14}$.

Cette clause circonstancielle sur la mémoire n'était pas nouvelle; elle était reprise de paix de religion antérieures. En 1563, l'édit d'Amboise demandait que toutes les occasions de discorde demeurent "éteintes, comme mortes, ensevelies«. La paix de Saint-Germain complétait sept ans plus tard: „Que mémoire de toutes choses passées d'une part et d'autre, et dès et depuis les troubles advenus notredit royaume, et à l'occasion d'iceux, demeure éteinte et assoupie comme de chose non advenue«. Moins d'un an après les massacres de la Saint-Barthélemy l'édit de Boulogne déclarait en juillet 1573 »Que la mémoire de toutes choses passées depuis le vingt-quatrième jour d'août demier passé, à l'occasion des troubles et émotions advenues en notre royaume, demeurera éteinte et assoupie, comme de chose non advenue«. Enfin, l'édit de Beaulieu, en mai 1576: „Que la mémoire de toutes choses passées d'une part et d'autre dès et depuis les troubles advenus en notre royaume, et à l'occasion d'iceux, demeurera éteinte et assoupie, comme de chose non advenue $^{15}$.

La Restauration, une paix de religion ${ }^{16}$ ? II ne s'agit certes pas de prétendre que les événements de la France au $\mathrm{XVI}^{\mathrm{e}}$ siècle aient informé la perception que les Anglais avaient de leur propre histoire dans les années 1640-1660 encore que cette piste ne soit pas nécessairement absurde. On avait précisément publié en 1647-1648 la version anglaise de l'»Histoire des guerres civiles de France« de 1'Italien Davila ${ }^{17}$. Que la révolution anglaise se soit terminée, en un sens, comme une guerre de religion n'implique pas qu'elle ait été pour autant une guerre de religion. Mais l'on ne saurait totalement rejeter cette présomption. Il existe une ressemblance au moins formelle entre les deux séries d'événements ${ }^{18}$. Le 25 octobre, Charles II usait de sa prérogative ecclésiastique, en tant que chef de l'Église nationale, pour rappeler qu'il avait eu l'occasion, durant son séjour sur le continent, de dialoguer avec des représentants des »meilleures Églises réformées de France, des Pays-Bas, et

14 Bernard CotTRET, 1598. L'édit de Nantes, Paris 1997, p 363.

15 Ibid., p. 84-86.

16 On renverra aussi ici aux analyses d'Olivier CHRISTIN, La paix de religion, Paris 1997.

17 H.C. Davila, The Historie of the Civill Warres of France, trad. de l'italien, Londres, W. Lee, D. Pakeman, G. Bedell, 1647-1648. Sur l'importance des guerres de religion de France pour les Anglais de la première modernité, voir John H.M. SALMON, The French Religious Wars in English Political Thought, Oxford 1959.

18 Voir à ce sujet l'intéressante analyse de John MORRILL, England's Wars of Religion, dans: ID. (dir.), The Nature of the English Revolution, Londres 1992, p. 33-44. 
d'Allemagne «19. Il évoquait aussi l'inquiétude qu'avait ressentie le monde protestant dans son ensemble face aux schismes et aux divisions qui avaient prévalu en Angleterre. Charles II, fidèle en cela aux idées antiques, médiatisées par la Renaissance, reconnaissait trois formes pures de la politique: la monarchie, l'aristocratie et la démocratie. Il énonçait enfin ce grand précepte que le gouvernement de l'État et celui de l'Église devaient suivre le même principe; ainsi on ne pouvait avoir une démocratie politique et une aristocratie dans l'Église. Non, il fallait que tous deux fussent monarchiques. L'épiscopat était à l'ordre ecclésiastique ce qu'était le roi à l'ordre civil.

La grande idée de concorde ecclésiastique était au centre de la Restauration. Elle échoua tristement. Une conférence se tint à Westminster, d'avril à juillet 1661, dans le quartier de la Savoy, au logis de l'évêque de Londres, Gilbert Sheldon ${ }^{20}$. Douze évêques étaient présents, ainsi que douze autres ministres, partisans de ce qui allait devenir le dissent. Douze, comme les apôtres. Mais cela ne suffit pas à apaiser les tensions, face à la vague de scrupules que suscitaient les rites de l'Église établie ${ }^{21}$. Les prières prescrites n'étaient-elles pas aussi valables que ces oraisons improvisées que recommandaient les puritains? "Si les saints pouvaient faire l'opinion, ou si l'opinion pouvait faire les saints, nous ne tarderions pas à avoir plus d'opinions que de saints dans ce pays«, observait-on benoîtement pour décrire les désordres de la période antérieure ${ }^{22}$. Il s'agissait désormais d'en revenir au »Book of Common Prayer«, fondement de la liturgie anglicane, remplacé, du moins en théorie car il s'était souvent maintenu en pratique, par le $»$ Directory for Public Worship« ${ }^{23}$. Richard Baxter représentait le point de

19 Charles II, A declaration to all His Majesties loving subjects, for the setling of church-government, Londres, R. Wood, 1660, p. 3.

20 Sheldon devenait archevêque de Cantorbéry en 1663. Il mourut en 1677.

21 A true and perfect copy of the whole disputation at the Savoy that was managed by the episcopal divines, as opponents, to prove that there is nothing sinful in the liturgy. Published to make intelligible the fragment already published by the Lord Bishop of Worcester, under the hands of Dr. Pierson, and Dr. Guning; and so much of his Lordships book against $\mathrm{Mr}$. Baxter, as concerneth that disputation, Londres, s.n., 1662.

22 Lawrence WOMOCK, Pulpit-Conceptions, Popular-Deceptions, or, The grand debate resumed, in the point of prayer wherein it appears that those free prayers so earnestly contended for have no advantage above the prescribed liturgie in publick administrations: being an answer to the Presbyterian papers presented to the most reverend the ls. bishops at the Savoy upon that subject, Londres, R. Royston, 1662. Voir C. DuRSTon, By the book or with the spirit. The debate over liturgical prayer during the English Revolution, dans: Historical Research 79, 203 (2006), p. 50-73.

${ }^{23}$ A Directory for publique worship of God throughout the three kingdoms of England, Scotland, and Ireland together with an ordinance of Parliament for the taking away of the Book of common-prayer, and for establishing and observing of this present directory throughout the kingdom of England and dominion of Wales, Londres, Company of Stationers, 1645. 
vue presbytérien. Il recommanda en vain d'ôter de la liturgie certaines prières ou certaines gestes à même de heurter les susceptibilités ${ }^{24}$. En particulier, il souhaitait extirper les restes de papisme de la liturgie, en y voyant des compromis provisoires, remontant à l'époque de la Réforme: »Nous souhaitons humblement que l'on considère que les réformateurs ont eu la sagesse [...] de varier aussi peu que possible des usages en cours chez les romanistes afin de gagner les papistes et de les amener à la communion de l'Église ${ }^{25}$. Parmi ces anciens compromis pédagogiques: les répons de l'assemblée au célébrant, la litanie, l'observance du carême, les fêtes des saints... Il fallait également laisser place à des oraisons improvisées, remplacer le mot de "prêtre « par celui, plus protestant, de "ministre«, et permettre au pasteur de ne pas porter de surplis. Ou de ne pas faire de signes de croix, en particulier lors du baptême. Cette volonté puritaine de purifier littéralement les rites se heurta aux usages accrédités et occasionna une rupture formelle entre les pasteurs adeptes du »Book of Common Prayer« et leurs adversaires. Les anglicans insistaient pour leur part sur les continuités, en évoquant la conférence de Hampton Court en 1604, qui avait bien rappelé le rôle des évêques: sans évêques, y aurait-il encore des rois? „No bishop, no king«, s'était exclamé justement Jacques $\mathrm{I}^{\text {er }}$. L'expérience n'avait pu qu'amplifier ce point de vue. En tout cas l'abolition de l'épiscopat et celui de la monarchie avaient marqué la guerre civile et la grande rébellion; leur réintroduction conjointe allait illustrer la Restauration. Il fallait rappeler la »supériorité« des évêques face au principe invoqué de la "parité« des pasteurs ${ }^{26}$. En 1662, l'acte d'Uniformité imposa à tous l'usage exclusif du »Book of Common Prayer « ${ }^{27}$. Ce n'était que la quatrième fois que la liturgie de l'Église d'Angleterre était prescrite par voie parlementaire ${ }^{28}$.

Guerre des clochers si l'on veut, mais qui se solda par le départ de l'Église nationale de près de deux milliers de ministres l'année suivante. Ceux-ci ne manquèrent pas d'adresser à leurs communautés respectives leurs sermons d'adieu, en insistant sur la »mort civile« à laquelle ils étaient condamnés ${ }^{29}$.

24 Richard BAXTER, An accompt of all the proceedings of the commissioners of both persuasions appointed by His Sacred Majesty, according to letters patent, for the review of the Book of common prayer, etc., Londres, s.n., 1661, p. 2.

25 Ibid., p. 3.

${ }^{26}$ Semper eadem, or, A reference of the debate at the Savoy, 1661 , to the conference at Hampton-Court, 1603/4 to which is added the petition of the then non-conformists: whereby it appears that what the Presbyterians \&c. now scruple at was satisfactorily and fully answer'd, Londres, W. Gilbertson, 1662, »To the Reader«, non paginé.

2713,14 Charles II, c. 4.

28 Act of Uniformity 1549 (2, 3 Edward VI, c 1), Act of Uniformity 1552 (5, 6 Edward VI, c. 1), Act of Uniformity 1559 (1 Eliz., c. 2).

29 A Collection of Farewel-Sermons preached by Mr. Calamy 1. Mr. Watson 2. Mr. Sclater 3. Mr. Watson 4. Dr. Jacomb 5. Mr. Case 6. Dr. Jacomb 7. Mr. Baxter 8. Mr. 
Ce requiem revêtit des accents pathétiques: les non-conformistes apparurent comme des victimes expiatoires, injustement chargées des péchés de la guerre civile et de la révolution. Certaines familles mêmes étaient divisées; ainsi le révérend Edmund Calamy (v. 1600-1666), ferme dans ses attaches presbytériennes, eut-il deux fils, Edmund (v. 1635-1685), fidèle à la confession de son père et Benjamin (1642-1686), défenseur acharné de l'anglicanisme. Benjamin Calamy publia même en français un "Conseil évangélique aux consciences scrupuleuses«, dans lequel on défendait, en prenant à témoin Jean Claude, le célèbre pasteur réformé français, la conformité ecclésiastique avec l'Église d'Angleterre:

Au moins est-il du devoir d'un chrétien d'être doux et paisible, de se conformer aux lois des magistrats, aux ordres établis par les puissances supérieures, et de maintenir l'unité et la concorde d'une Église. Si les coutumes et les constitutions d'un pays sont défendues de Dieu, il faut s'en abstenir et plutôt sacrifier sa vie; sinon, Dieu même nous oblige expressément à l'obéissance et à la soumission ${ }^{30}$.

Voulez-vous renforcer une cause? Persécutez-la. Les dissenters firent l'objet d'une exclusion programmée qui renforça en réalité la pugnacité des plus décidés. Connues sous le nom de Clarendon Code, du nom du principal ministre de Charles II à l'époque, ces vexations prenaient des formes absurdes: défense de siéger au sein des municipalités, défense faite aux pasteurs et aux maîtres d'école de se rapprocher de leurs anciennes églises, ou encore interdiction des "conventicles«, ou assemblées religieuses illicites... La nonconformité ecclésiastique allait-elle équivaloir à une mort civile? Les dissenters se dotèrent sans attendre de leurs martyrologes ${ }^{31}$ : le christianisme persécuteur n'a pas longtemps une très bonne presse. De plus, les frontières entre conformité et non-conformité se révélèrent bien poreuses à l'usage, et les aller et retour entre l'Église établie et les communautés dissidentes défrayèrent la chronique pendant plusieurs décennies. Le pasteur anglo-normand Jean Durel tenta de freiner l'hémorragie en déclarant que les Églises réformées du continent n'entretenaient que de lointains rapports avec les communautés dissidentes de Grande-Bretagne. Il publiait à cet effet une série d'extraits de lettres démontrant que la doctrine anglicane était jugée parfai-

Jenkins 10. Mr. Lye 11. 12. Dr. Manton 13. To their respective congregations at their departure from them, s.l. 1662.

30 Conseil evangelique aux consciences scrupuleuses ou sermon sur ces paroles de Jesus Christ mais plus-tôt donnez en aumônes ce que vous avez, et voicy toutes choses vous seront nettes. St. Luc. c. 11, v. 41, Londres, G. Wells, 1683, „épître dédicatoire du traducteur«, non paginée.

31 Citons entre autres exemples, A Brief narration of the imprisonment of Mr. Francis Bampfield [...] with Mr. Phillips (his assistant) being now both turn'd out for their non-conformity: as also the imprisoning of several of the inhabitants who came to hear him exercise at his own house, September the 19, 1662, s.l. 1662. 
tement saine par les protestants français du continent ${ }^{32}$. Les non-conformistes étaient une épine dans le pied de la société anglaise; mais ils pouvaient aussi se révéler utiles à la monarchie, qui comptait paradoxalement sur leur appui pour affirmer son pouvoir. Le 26 décembre 1662, Charles II avait insisté sur le caractère providentiel de son retour au trône pour prêcher la concorde:

Comme il a plu à Dieu de nous restaurer merveilleusement sur le trône de nos ancêtres et d'accorder à nos sujets la paix et la tranquillité, sans que le glaive ne verse le moindre sang, voulant leur sécurité sans que l'épée de justice ne s'appesantisse sur quiconque, nous souhaitons convaincre les récalcitrants par notre clémence, au lieu de les punir par la rigueur de la loi ${ }^{33}$.

Le roi reconnaissait que certaines exécutions avaient été nécessaires, faisant ainsi allusion au procès des régicides, mais, ajoutait-il, wnous voulons appliquer les antidotes requis aux insinuations vénéneuses par lesquelles certains de nos sujets endurcis, nous dit-on, tentent d'empoisonner notre peuple, en trompant les entendements « $^{34}$. Et Charles II de confirmer sa déclaration de Breda. Mais le roi avouait qu'il ne pouvait accorder cette liberté de conscience aux dissenters qu'en utilisant sa prérogative, et non en obtenant une loi du Parlement, que l'on devine plus rétif que le monarque à admettre la moindre dérogation à l'uniformité ecclésiastique:

En ce qui concerne les peines encourues par ceux qui, tout en vivant de façon pacifique, ne se conforment pas, et invoquent leur conscience erronée, mais vaquent à leurs dévotions sans le moindre scandale, nous nous engageons personnellement, sans enfreindre les libertés du Parlement, à en convaincre les membres d'adopter une loi en ce sens, nous réservant la possibilité d'utiliser le pouvoir de dispenser qui nous appartient en propre35.

En l'absence de législation appropriée, le roi pouvait-il dispenser les nonconformistes des peines encourues? Ce pouvoir de dispenser quiconque des rigueurs de la loi et de suspendre l'application des lois allait être revendiqué avec force par la couronne sous la Restauration ${ }^{36}$. Il correspondait à une urgence réelle: comment condamner impunément toute une partie de la population à l'illégalité? D'autant plus que cette illégalité concernait l'exercice du

32 John DUREL, The liturgy of the Church of England asserted in a sermon preached at the chappel of the Savoy, before the French congregation, which usually assembles in that place, upon the first day that divine service was there celebrated according to the liturgy of the Church of England, Londres, R. Royston, 1662.

${ }^{33}$ His Majesties declaration to all his loving subjects, December 26, 1662, Londres, J. Bill, C. Barker, 1662, p. 1.

34 Ibid., p. 2.

35 Ibid., p. 8.

36 C.A. EDIE, Tactics and Strategies. Parliament's Attack upon the Royal Dispensing Power 1597-1689, dans: The American Journal of Legal History 29/3 (1985), p. 197234. 
culte. Charles II souhaitait du reste étendre cette tolérance aux catholiques et ne pas s'en tenir aux seuls non-conformistes protestants: "Nous n'admettrions pas que l'on mette à mort certains de nos sujets pour des questions d'opinion en matière religieuse uniquement $\ll^{37}$.

La politique d'indulgence reprit brièvement dans les années 1670: il s'agissait pour le trône de se poser en souverain arbitre, et ces hommes que l'on redoutait officiellement et dans lesquels on voyait des républicains potentiels servirent de faire-valoir aux souverains Stuarts, toujours prêts à quelque bonne action qui renforçât leur pouvoir absolu. Quoi de plus beau que la clémence d'Auguste? Quoi de plus auguste que l'indulgence? Tout comme l'aumône renforce la satisfaction d'être riche, l'indulgence royale était une façon habile d'affirmer la prérogative du souverain. Cette indulgence fut d'abord un échec en 1672-1673. Elle faillit réussir en 1687-1688. Et pourtant, Charles II ne manquait pas d'arguments à faire valoir en faveur de sa politique en mars 1672 . Il commençait par rappeler l'absence d'efficacité de la coercition en matière de conscience: on peut croire, ou ne pas croire, mais on ne saurait obliger à croire. Et tout en affirmant son attachement à l'Église nationale, Charles II expliquait que son bon plaisir était que l'application des lois pénales contre les non-conformistes protestants ou catholiques fût suspendue $^{38}$. Peine perdue. En dépit d'une trêve dans l'application des rigueurs de la loi, le pays n'était pas prêt à admettre le pluralisme religieux. Le Parlement répliqua en passant deux Test Acts exigeant en mars 1673 que l'on communiât selon le rite anglican avant d'exercer tout emploi public ${ }^{39}$. Pour un catholique, une telle communion eût constitué un sacrilège. Mais la mesure était moins efficace contre les non-conformistes protestants, immédiatement accusés de dissimulation et de "conformité occasionnelle«.

La politique d'indulgence allait être reprise sous le règne suivant. En avril 1687, Jacques II publiait sa Déclaration pour la liberté de conscience.

Comme il a plu à Dieu, non seulement de nous amener à la couronne impériale de ces royaumes, au milieu des plus grandes difficultes, mais de nous maintenir par sa providence plus qu'ordinaire sur le trône de nos royaux ancêtres, il n'y a rien que nous désirions plus désormais que d'établir notre gouvernement sur des fondements qui garantissent le bonheur de nos sujets, et qui les unissent à nous par inclination comme par devoir; rien ne saurait mieux le garantir désormais que le libre exercice de leur religion, auquel on ajoutera la parfaite jouissance de leurs biens, qui a toujours été respectée par nous depuis que nous portons la couronne. Ce sont là les deux choses

37 His Majesties declaration to all his loving subjects, December 26, 1662, Londres, J. Bill, C. Barker, 1662, p. 11.

38 His Majesties declaration to all his loving subjects, March 15. 1672. Published by the advice of his Privy Council, Edimbourg, E. Tyler, 1672.

3925 Car. II, c. 2. La mesure fut renforcée en 1678 (30 Car. II, 2, c. 1). 
qui comptent le plus aux yeux des hommes, et elles seront garanties dans ces royaumes, tant que nous régnons, afin de préserver la paix et notre gloire 40 .

Alors que son frère Charles II s'était converti au catholicisme sur son lit de mort, Jacques II ne craignait pas de professer son attachement à la foi romaine. Le petit-fils de Henri IV, le fils de Henriette-Marie et de Charles ${ }^{\text {er }}$, le roi martyr, s'était converti à la religion catholique, sans craindre d'endurer bien des avanies. Le Test Act était dirigé en grande partie contre lui alors qu'il n'était encore que duc d'York. En 1687, alors qu'il occupait le trône depuis moins de deux ans, il se décida à agir, convaincu sans doute que Dieu était avec lui. L'expression de providence extraordinaire - notons la litote "providence plus qu'ordinaire « - désigne cette attente du miracle permanent, ou du moins de la grâce, susceptible selon Jacques II de ramener son royaume dans l'orbite de la foi romaine, pourtant abhorrée par nombre de ses compatriotes. La révocation de l'édit de Nantes par Louis XIV pesait d'un poids fort lourd dans cette image négative ${ }^{41}$. Jacques II, comme il le soulignait lui-même, occupait la couronne simpériale« des trois royaumes encore distincts d'Angleterre, d'Écosse et d'Irlande. Cet imperium s'entendait de façon plus ecclésiastique que territoriale; nul impérialisme en tout cela, mais l'idée que le roi, comme l'avait dit l'adage, était »empereur en son royaume«. Depuis Henry VIII, aucun pays d'Europe qui n'exerçât cette plenitudo potestatis avec plus d'assiduité que l'Angleterre. Un roi d'Angleterre se voyait confier l'intégralité des pouvoirs politiques et religieux. C'est parce qu'il était à la tête d'une Église nationale protestante que, bien que catholique, Jacques II pouvait ordonner à ses évêques de lire en chaire sa déclaration. Situation paradoxale, mais qui avait été celle du retour du royaume au catholicisme sous Mary Tudor au siècle précédent. Jacques II ne rompait pas pour autant avec l'esprit de la Restauration de 1660, comme Charles Ir avant lui il rappelait son attachement à une politique d'indulgence et au respect scrupuleux des biens de ses sujets. Liberté et propriété allaient être durablement les principaux articles du credo politique des Anglais. Jacques II ne craignait pas pour autant de réaffirmer que son vœu le plus cher aurait été que tous les Anglais fussent catholiques comme lui. Si les rois et ses sujets avaient partagé la même religion, cela eût garanti une communauté d'inclination favorable à la paix civile; mais sans vouloir précipiter les choses, Jacques II prenait acte de l'échec de la politique de conformité ecclésiastique. Et rappelant son refus de la contrainte ecclésiastique, il suspendait provisoirement les lois pénales contre catholiques et dissenters, en attendant que le Parlement le suivît sur

40 His Majesties gracious declaration to all his loving subjects for liberty of conscience, Londres, C. Bill, H. Hills, T. Newcomb, 1687.

41 Bernard COTTRET, Glorreiche Revolution, schändliche Revokation?, dans: Rudolf von ThadDEN, Michelle MAGDELAINE (dir.), Die Hugenotten, Munich 2005, p. 7384. 
cette voie. Désormais, par sa seule autorité, Jacques II déliait un certain nombre de ses sujets de la loi.

Cette prérogative régalienne n'était pas totalement nouvelle en droit anglais, bien que l'on tentât évidemment de la présenter comme une innovation inadmissible. Le mot innovation avait une valeur purement négative à l'époque. Réciproquement, les défenseurs de la prérogative tentèrent de démontrer que le plus glorieux des règnes, celui de la reine Elisabeth, n'avait pas ignoré la pratique ${ }^{42}$. Il s'agissait de démontrer que le pouvoir de dispenser n'avait aucun caractère »arbitraire« puisqu'il s'agissait à l'inverse pour Jacques II de pourfendre l'esprit de persécution. Le pouvoir de dispenser était même l'un des »joyaux de la couronne«:

La dispense est plus que l'interprétation, mais moins que l'abrogation; par un acte volontaire émanant de la grâce et de la faveur du prince, une personne ou une communauté est déliée de l'obéissance à une loi qui continue à s'appliquer pour tous ceux qui n'ont pas fait l'objet de cette dispense ${ }^{43}$.

Et comme il fallait appuyer cette analyse sur un précédent historique, l'auteur citait un acte de l'époque de Henry VII, né des incertitudes de la guerre des Deux-Roses et de son règlement ${ }^{44}$. En avril 1688 , Jacques II reprenait sa déclaration, en évoquant le soutien de nombreux non-conformistes protestants ${ }^{45}$. La résistance des évêques, le débarquement de Guillaume d'Orange, la fuite de Jacques II s'ensuivirent. On a pu qualifier cet événement de »Glorious Revolution«. La couronne fut confiée conjointement à Guillaume et à son épouse, Mary, fille de Jacques II. Ces événements sont bien connus, et l'on prendra le parti de ne pas les reprendre dans leur intégralité. Reste la question de leur interprétation. La Glorious Revolution marqua-t-elle la fin de la période de la Restauration? Ou bien en constitua-t-elle la répétition?

42 An instance of Queen Elizabeth's power of dispensing with Acts of Parliament: offer'd to the consideration of the gentlemen of the University of Cambridge. Together with some queries thereupon, Londres, H. Hills, 1687.

43 The King's dispensing power explicated and asserted, Londres. R. Janeway, 1687, p. 2.

44 Cet acte de 1495 laissait au roi le soin d'apprécier si un homme qui lui aurait été fidele tout en niant la validité de son pouvoir n'était pas dispensé de la rigueur de la loi contre la haute trahison (11 Henry VII, c. 1). L'on pouvait donc distinguer entre deux types de maux: malum per se et malum prohibitum; il revenait au roi d'apprécier s'il fallait punir ce qui relevait uniquement de la seconde catégorie.

45 His Majesties gracious declaration to all his loving subjects for liberty of conscience, Londres, C. Bill, H. Hill, T. Newcomb, 1688. 


\section{La tolérance contre l'indulgence}

Il appartenait à la Glorious Revolution de porter un terme définitif à la révolution des années 1642-1660. Et à l'interrègne cromwellien. La société anglaise tout entière était appelée à s'unir derrière Guillaume et Mary, et à panser ses plaies grâce à deux actes fondateurs, le Bill of Rights, et la »loi sur la tolérance« - ainsi qu'on l'appelle communément. Nous ne reviendrons pas sur le Bill of Rights, ni sur le Heads of Grievances plus radical qui l'avait précédé46. Un terme était mis à la possibilité pour la couronne de suspendre des lois - ou de dispenser quiconque de leur application. Le règlement ecclésiastique intervenait grâce à un vacte exemptant les sujets protestants de Leurs Majestés séparés de l'Église d'Angleterre des peines encourues à la suite de certaines lois«. Il s'agissait, comme précédemment, de permettre la liberté de conscience, mais contrairement à la politique d'indulgence menée par les Stuarts, et qui s'est souvent prêtée au sarcasme, cet acte magnanime est passé à la postérité sous le nom philosophique de »loi sur la tolérance«. Or le mot ne figure pas, et l'on trouve un texte alambiqué, dont le principal mérite est d'avoir été inapplicable. Pour avoir droit à cette liberté de conscience, il fallait souscrire à une déclaration contre le Saint-Siège, évidemment destinée à exclure davantage les catholiques anglais, tout en admettant la Trinité ou l'inspiration de la Bible ${ }^{47}$. L'ambiguité n'est pas un défaut; il lui arrive même d'être une vertu lorsque les valeurs du consensus l'emportent sur toute autre considération.

Il n'y avait rien là de très philosophique. Mais le mot »tolérance« avait été popularisé par John Locke, et on s'empressa de conférer à l'ensemble de la Glorious Revolution un caractère philosophique qu'elle n'avait certes pas à l'origine ${ }^{48}$. Restait l'épineuse question de la légitimité du pouvoir. En particulier, les ecclésiastiques qui avaient prêté serment d'obéir à Jacques II pouvaient-ils reporter leur allégeance sur Guillaume, devenu opportunément Guillaume III? Un schisme s'ensuivit dans l'Église anglicane. Décidément, les fractures religieuses poursuivaient la société anglaise. Certains hommes d'Église se demandaient, avec angoisse, si l'on pouvait prêter serment à Guillaume après avoir juré fidélité à Jacques. William Sancroft, l'archevêque de Cantorbéry, était destitué; il s'était pourtant opposé à la politique religieuse de Jacques II, mais sans pour autant admettre l'usurpateur Guillaume. On

46 Bernard CotTret, Michael Hearn, Michel Lemosse et al. (dir.), Histoire du Royaume-Uni. Une anthologie, Rosny 2001, p. 155-158.

47 Bernard CotTret, La Glorieuse Révolution d'Angleterre, 1688, Paris 1988, p. 142-145.

48 Ce rôle éclairé de la Glorieuse Révolution a été bien analysé récemment par HansChristof KraUs, Englische Verfassung und politisches Denken im Ancien Régime 1689 bis 1789 , Munich 2006. 
baptisa du nom de »non-jureurs« ces séparatistes: neuf évêques, quatre cents pasteurs, quelques Écossais et un évêque d'Irlande firent sécession. Une Église anglicane séparée devait exister jusqu'à la fin du XVIII ${ }^{\mathrm{e}}$ siècle.

Un non-jureur n'était pas pour autant un révolutionnaire. Le nouvel archevêque de Cantorbéry, Edward Stillingfleet, défendait le droit pour la couronne de nommer de nouveaux évêques à la place du clergé non-jureur ${ }^{49}$. William Sherlock appelait à reconnaître Guillaume, en expliquant qu'après avoir refusé de prêter allégeance au nouveau roi il avait été convaincu par des arguments contraires: "J'ai prié Dieu de tout mon coeur de me dire si je me trompais, avant de quitter mon ministère sur une simple erreur, et, Dieu merci, j'ai reçu la satisfaction que j'attendais $^{50}$. Sherlock expliquait, citant l'apôtre Paul, qu'il fallait toujours respecter les détenteurs de l'autorité, quand bien même leurs titres eussent été mal assurés. "Que toute personne soit soumise aux autorités supérieures; car il n'y a point d'autorité qui ne vienne de Dieu, et les autorités qui existent ont été instituées par Dieu«, avait déclaré l'auteur de l'épître aux Romains ${ }^{51}$. Peu convaincu par les arguments de son confrère, le révérend John Kettlewell répondait à Sherlock, et il rappelait à l'usage de ses frères non-jureurs comme lui que le christianisme conduisait à la croix ${ }^{52}$. Ou que les persécutés confessaient souvent la vérités3. Il mourut non sans trouver le temps de composer un ars moriendi ${ }^{54}$. Les non-jureurs étaient les représentants d'un courant dévot interne à l'anglicanisme, qui s'attira souvent

49 Edward StILLnNGrLeET, A Vindication of Their Majesties Authority to Fill the Sees of the Deprived Bishops, Londres, R. Chiswell, 1691.

so William SHERLOCK, The case of allegiance due to soveraign powers stated and resolved, according to Scripture and reason, and the principles of the Church of England, with a more particular respect to the oath, lately enjoyned, of allegiance to their Their present Majesties, K. William and Q. Mary, Londres, W. Rogers, 1691, préface, non paginée.

51 N.T. Rom 13, 1.

52 John KETTLEWELL, The duty of allegiance settled upon its true grounds, according to Scripture, reason, and the opinion of the Church in answer to a late book of Dr. William Sherlock, master of the Temple, entituled, The case of the allegiance due to sovereign powers, stated, and resolved, according to Scripture, \&c.: with a more particular respect to the oath lately injoyn'd, Londres, [s.n.] 1691; ID., Christianity, a doctrine of the cross, Londres, J. Hindmarsh, R. Kettlewell, 1691.

53 ID. A companion for the persecuted, or, An office for those who suffer for righteousness containing particular prayers and devotions, for particular graces, and for their private or publick wants and occasions, Londres, s. n., 1693.

54 ID., Death made comfortable, or, The way to dye well consisting of directions for an holy and an happy death: together with an office for the sick and for certain kinds of bodily illness, and for dying persons, and proper prayers upon the death of friends, Londres, R. Kettlewell, 1695. 
les quolibets de ses opposants, qui dénonçaient la tartufferie de ces protestants, toujours suspects de ressembler secrètement aux catholiques abhorrés ${ }^{55}$.

\section{Le temps des usurpations}

Dans l'histoire anglaise, la distinction paulinienne entre les pouvoirs légitimes et illégitimes, tous à leur façon institués par Dieu, prenait un relief singulier. Ce pragmatisme britannique a longtemps surpris les continentaux. En 1714, par exemple, au lendemain de la paix d'Utrecht et à la veille de la disparition de Louis XIV paraissait en France un ouvrage destiné à lever le trouble dans les esprits sous le titre d'»Histoire succincte de la succession à la couronne de la Grande-Bretagne depuis le commencement de la monarchie jusqu'à présent«. 1714, c'était également l'année où Georges I ${ }^{\text {er }}$ de Hanovre accédait au pouvoir, en lieu et place du prétendant Stuart, disqualifié par son attachement au catholicisme romain.

La succession à la couronne de la Grande-Bretagne faisant aujourd'hui un des plus grands sujets d'entretien, déclarait l'auteur, il n'y a aucun doute qu'on aspire à une histoire qui faisant voir clairement et succinctement sur la tête de qui elle a été mise et pour quelles raisons, et sur quels fondements le cours naturel de la succession a été détourné, puisse nous instruire de ce qu'on a cru dans tous les siècles au sujet d'un point si débattu dans le temps présent, et partant nous mette en état d'en bien juger ${ }^{56}$.

L'on y reprenait les analyses d'un non-jureur, le révérend George Harbin, qui s'était encore interrogé de façon toute récente sur les ressorts de la légitimité dynastique. Harbin avait rappelé le caractère héréditaire de la succession, contre William Higden, un ancien non-jureur retourné, comme Sherlock avant lui. Higden avait expliqué que rien ne distinguait à la limite la possession de facto de la possession de jure de la couronne ${ }^{57}$. Harbin ne pouvait pas l'admettre:

C'est du temps des usurpations qui succédèrent à la mort de Charles I ${ }^{\text {er }}$ que l'on commença en ce royaume à prêter allégeance aux pouvoirs en place quels qu'ils fussent. Dans toutes les révolutions qui avaient précédé, les princes qui prenaient possession de la couronne invoquaient le droit et déclaraient la posséder de droit. Mais le Parlement croupion, Cromwell et les usurpateurs qui s'ensuivirent ne pouvaient en aucune

55 Colley Cibber, un temps directeur du Theatre Royal Drury Lane, à Londres, mit à l'affiche en 1717 une adaptation du "Tartuffer de Molière sous le titre évocateur de »The Nonjuror

56 Histoire succincte de la succession à la couronne de la Grande-Bretagne, s.l. 1714, p. 3.

57 William Higden, A View of the English Constitution, With Respect to the Sovereign Authority of the Prince, and the Allegiance of the Subject, Londres 1709. 
façon le prétendre, et leurs partisans prétendirent que le fait faisait droit; plusieurs livres furent publiés par des papistes, des fanatiques et des déistes à l'appui de cette thèse. Mais les fidèles de l'Église d'Angleterre généralement rejetaient ce principe, et beaucoup de presbytériens étaient d'accord avec eux pour dire que l'on devait prêter allégeance au roi légitime, même quand il avait été chassé, et nombre d'auteurs des deux confessions écrivirent contre le pouvoir de fait ${ }^{58}$.

De Guillaume le Conquérant en 1066 à Guillaume d'Orange en 1688, en passant par la guerre des Deux-Roses, la question de la légitimité en Angleterre avait connu bien des aléas ${ }^{59}$. Comment distinguer désormais le droit du fait du fait du droit?

58 George HARBIN, The Hereditary Right of the Crown of England Asserted, Londres, R. Smith, 1713, p. 1.

59 Bernard CotTret, Histoire de l'Angleterre. De Guillaume le Conquérant à nos jours, Paris 2007. 
